\title{
La violencia familiar en la sierra central del Perú. Caso: Marco, Tunanmarca y Acolla
}

\author{
Family violence in the central sierra of Peru \\ Case: Marco, Tunanmarca and Acolla
}

\author{
Eugenia Fabian A.!' Luz M. Vilcas B.!'y Nidia G. Matos M!!! \\ (1) Facultad de Trabajo Social, Universidad Nacional del Centro del Perú \\ Email: eugefabi12@gmail.com
}

\section{Resumen}

El objetivo del presente estudio fue conocer cómo se manifiesta la violencia familiar en los distritos de Marco, Acolla y Tunanmarca de la provincia de Jauja, el nivel de investigación es descriptiva, el tipo es básico, diseño de investigación no experimental transversal, descriptiva, el carácter de la investigación es mixta, la población en estudio es aproximadamente 200 familias, que sufren violencia y la muestra es no probabilística de 30 casos que afrontan problemas de violencia familiar y para la parte cualitativa, se ha considerado, solo 9 casos, siendo tres casos por distrito. El método que se utilizo fue el análisis- síntesis, los instrumentos fueron el cuestionario para la parte cuantitativa y la guía de entrevista para la parte cualitativa, las técnicas fueron la encuesta y la entrevista respectivamente. El resultado del estudio la violencia física a la mujer de parte del cónyuge algunas veces atraves de empujones, patadas, pelliscones, sacudidas y/o jaloneos que oscila Marco del 7\% al 54\%, en Acolla del 3\% al 47\% y en Tunanmarca del 3\% al 50\%; $L$ a violencia verbal en Marco ocurre a través de insultos, gritos y acusaciones algunas veces lo que oscila del $7 \%$ al $47 \%$, en Acolla del 3\%AL 36\% y en Tunanmarca del 17 al 33\% y en cuanto a la violencia psicológica también es algunas veces a través de amenazas y humillaciones y no existe violencia sexual. Por otra parte, la violencia de padres a hijos es también algunas veces atraves de la violencia verbal, física y psicológica los que oscilan en Marco del $3 \%$ al 34\%, En Acolla del 3\% al 24\% y en Tunanmarca del $17 \%$ al 34\%. En conclusión, la violencia en las familias de Marco, Acolla y Tunanmarca es algunas veces fundamentalmente a través de la violencia verbal, seguido por la físico y psicológico y no existe violencia sexual, así mismo la violencia de padres a hijos es solo algunas veces como medio de corrección en la buena formación.

Palabras Claves: violencia familiar, violencia verbal, violencia física, violencia sexual

\section{Abstract}

The objective of the present study was to know how family violence manifests itself in the districts of Marco, Acolla and Tunanmarca of the province of Jauja, the level of research is descriptive, the type is basic, cross-sectional, descriptive, descriptive research design, The nature of the research is mixed, the population under study is approximately 200 families, who suffer violence and the sample is not probabilistic of 30 cases that face problems of family violence and for the qualitative part, it has been considered, only 9 cases, being three cases by district. The method that was used was the analysis-synthesis, the instruments were the questionnaire for the quantitative part and the interview guide for the qualitative part, the techniques were the survey and the interview respectively. The result of the study the physical violence to the woman on the part of the spouse sometimes through pushing, kicking, pelliscones, jerks and / or jerks that oscillates Marco from 7\% to 54\%, in Acolla from 3\% to 47\% and in Tunanmarca from 3\% to 50\%; Verbal violence in Marco occurs through insults, shouts and accusations sometimes ranging from $7 \%$ to $47 \%$, in Acolla from 3\% to $36 \%$ and in Tunanmarca from 17 to $33 \%$ and in terms of psychological violence it is also sometimes through threats and humiliations and there is no sexual violence. On the other hand, violence from parents to children is also sometimes through verbal, physical and psychological violence ranging from 3\% to 34\% in Marco, 3\% to 24\% in Acolla and 24\% in Tunanmarca to 3. 4\%. In conclusion, the violence in the families of Marco, Acolla and Tunanmarca is sometimes fundamentally through verbal violence, followed by physical and psychological violence and there is no sexual violence, so violence from parents to children is only sometimes correction medium in good training.

Keywords: family violence, verbal violence, physical violence, sexual violence 


\section{Introducción}

La violencia y el maltrato en el ámbito familiar se reconocieron como un problema social únicamente a partir de 1960, cuando algunos autores descubrieron el síndrome del niño maltratado, antes de esa fecha la violencia contra el género femenino era considerado como un fenómeno poco frecuente estaba catalogado como anormal y se le atribuía a personas con trastornos psicopatológicos, Alvarado, Salvador, Estrada y Terrones (1998).

Entre las conclusiones del estudio de la OMS se destaca que la violencia contra las mujeres mostraba una gran diversidad en diferentes entornos. El porcentaje de mujeres que habían sufrido violencia física a manos de sus parejas oscilaba entre el 13\% en Japón y el $61 \%$ en el entorno rural del Perú. En una revisión de 50 estudios realizados alrededor del mundo, la violencia varía entre un $10 \%$ a un $50 \%$ de mujeres que informan haber sido golpeadas o heridas por sus compañeros en algún momento de sus vidas. (Heysi etal; 1999) citado por Parra $(20 \mid 2)$.

La prevalencia a nivel mundial de la violencia hacia la mujer por su pareja se ha elevado en comparación con la década de los noventa, según El Banco Mundial, la OMS, la Unión Africana y la Comisión de los derechos humanos que alcanza del 10 al 69\%.López $(20 \mid 1)$.

Perú es un país latinoamericano, como afirman (Morrison y Biehi, 1999) Latinoamérica es una de las regiones más violentas del mundo en las calles y los hogares. Es de notar que la violencia en las calles tiene la dirección hombre a hombre, mientras que la violencia en el Hogar la tiene de hombre a mujer.

La prevalencia de la violencia en el hogar en las zonas rurales en muchos casos sobrepasa al de los

latinos (veinte veces) mientras que en el contexto social andino es considerablemente menos violento que el latino de acuerdo a lo que manifiestan las propias víctimas. El objetivo central del estudio fue conocer cómo se manifiesta la violencia familiar en Marco, Acolla, Tunanmarca, la hipótesis general fue que la violencia familiar se manifiesta algunas veces, a través de la violencia física, verbal, psicológica y sexual.

\section{Materiales y métodos}

El tipo de investigación es básico, nivel descriptivo, diseño no experimental, transversal descriptivo. Los instrumentos de recolección de datos, fue el cuestionario para el estudio cuantitativo y la guía de entrevista para el cualitativo. La población en estudio de los distritos de Marco Acolla y Tunan Marca de la provincia de Jauja, fue aproximadamente 200 familias, que sufren violencia en los distritos mencionados y la muestra fue de 30 casos que afrontan problemas de violencia familiar en Marco, Tunan Marca y Acolla para la parte cuantitativa. Para la parte cualitativa, se ha considerado, solo 9 casos, siendo tres casos por distrito. El método que se utilizo fue el análisis- síntesis.

Los procedimientos de recolección de datos fueron: Revisión de la bibliografía relacionada al tema de investigación. Observación de la realidad cotidiana de las familias del distrito de Marco, Acolla y Tunanmarca.

Entrevista a las autoridades líderes y pobladores de los distritos mencionados con el propósito de identificar los casos de violencia familiar. (Violencia a la mujer de parte del cónyuge y violencia de padres a hijos).

Aplicación de los cuestionarios a las familias identificadas y entrevistas a los casos detectados.

\section{Resultados}

Tabla I.Violencia física conyugal en marco, Acolla y Tunanmarca

\begin{tabular}{|c|c|c|c|c|c|c|c|c|c|c|c|c|c|c|c|c|c|c|c|}
\hline \multirow{3}{*}{$\begin{array}{l}\mathrm{N} \\
0\end{array}$} & & \multicolumn{6}{|c|}{ MARCO } & \multicolumn{6}{|c|}{ ACOLLA } & \multicolumn{6}{|c|}{ TUNAN MARCA } \\
\hline & \multirow{2}{*}{$\begin{array}{l}\text { REACTIVO } \\
\text { S }\end{array}$} & \multicolumn{2}{|c|}{ NUNCA } & \multicolumn{2}{|c|}{$\begin{array}{l}\text { ALGUNAS } \\
\text { VECES }\end{array}$} & \multicolumn{2}{|c|}{ SIEMPRE } & \multicolumn{2}{|c|}{ NUNCA } & \multicolumn{2}{|c|}{$\begin{array}{l}\text { AGUNAS } \\
\text { VECES }\end{array}$} & \multicolumn{2}{|c|}{ SIEMPRE } & \multicolumn{2}{|c|}{ NUNCA } & \multicolumn{2}{|c|}{$\begin{array}{l}\text { AGUNAS } \\
\text { VECES }\end{array}$} & \multicolumn{2}{|c|}{ SIEMPRE } \\
\hline & & $\mathrm{N}^{\circ}$ & $\%$ & $\mathrm{~N}^{\circ}$ & $\%$ & $\mathrm{~N}^{\circ}$ & $\%$ & $\mathrm{~N}^{\circ}$ & & $\mathrm{N}^{\circ}$ & $\%$ & $\mathrm{~N}^{\circ}$ & $\%$ & $\mathrm{~N}^{\circ}$ & $\%$ & $\mathrm{~N}^{\circ}$ & $\%$ & & $\%$ \\
\hline 1. & Alguna vez te ha empujado tu & 21 & 70 & 4 & 21 & 5 & 17 & 24 & 80 & 6 & 20 & 0 & 0 & 26 & 87 & 4 & 13 & 0 & 0 \\
\hline 2. & $\begin{array}{l}\text { Te ha golpeado con un objeto o te } \\
\text { ha lanzado cosas cuando se } \\
\text { enojan o discuten. }\end{array}$ & 23 & 76 & 5 & 17 & 2 & 7 & 19 & 63 & 6 & 20 & 5 & 17 & 20 & 66 & 5 & 17 & 5 & 17 \\
\hline 3. & Te ha destruido algunas cosas. & 29 & 97 & 1 & 3 & 0 & 0 & 25 & 83 & 5 & 17 & 0 & 0 & 28 & 97 & 2 & 3 & 0 & 0 \\
\hline 4. & Te ha pateado. & 19 & 64 & 10 & 33 & 1 & 3 & 24 & 80 & 6 & 20 & 0 & 0 & 21 & 70 & 8 & 27 & 1 & 3 \\
\hline 5. & Te ha sacudido o jaloneado. & 10 & 33 & 16 & 54 & 4 & 13 & 15 & 50 & 14 & 47 & 1 & 3 & 14 & 47 & 15 & 50 & 1 & 3 \\
\hline 6. & Te pellizca en el cuerpo o tus & 21 & 70 & 8 & 27 & 1 & 3 & 24 & 80 & 6 & 20 & 0 & 0 & 20 & 66 & 8 & 27 & 2 & 7 \\
\hline 7. & Le tira al suelo y le da patadas & 28 & 93 & 2 & 7 & 0 & 0 & 29 & 96. & 1 & 3 & 0 & 0 & 29 & 97 & 1 & 3 & 0 & 0 \\
\hline 8. & $\begin{array}{l}\text { Le golpea tan fuerte hasta llevarla } \\
\text { al médico. }\end{array}$ & 30 & 100 & 0 & 0 & 0 & 0 & 30 & $\begin{array}{l}10 \\
0\end{array}$ & 0 & 0 & 0 & 0 & 30 & 100 & 0 & 0 & 0 & 0 \\
\hline
\end{tabular}

Fuente: Cuestionario aplicado por las investigadoras a los padres e hijos de Marco en Mayo del 2015 
En el cuadro $\mathrm{N}^{\circ} \mathrm{O}$ l, se observa la violencia física conyugal en los distritos de Marco, Acolla y Tunanmarca, los porcentajes más altos de los tres distritos corresponde al ítem nunca, es decir que el alto porcentaje de ellos nunca han sufrido violencia como es en el caso de Marco oscila del 33\% al 93\% de mujeres que nunca han sufrido violencia física de parte de su conyugue; en Acolla de acuerdo a la opinión de las propias mujeres nunca han sufrido violencia del 50\% al 96\% ; así mismo en Tunanmarca nunca han sufrido violencia un porcentaje que oscila del $47 \%$ al $97 \%$. En el ítem algunas veces sufrieron violencia en Marco del 7\% al 54\%, en Acolla del $3 \%$ al $47 \%$ y en Tunan Marca del $3 \%$ al $50 \%$ y en el Ítem siempre se percibe que en los tres distritos los porcentajes más bajos oscilan del 3\% al 17\% .Como se puede observar la violencia física conyugal en los distritos mencionados son similares, no se tiene diferencias significativas.

Es decir la violencia física hacia la mujer de parte del cónyuge se manifiesta a través de patadas, pellizcones, jaloneos y lo sacudidas y empujones ocurre por lo general solo algunas veces.

Tabla 2. Violencia verbal conyugal

\begin{tabular}{|c|c|c|c|c|c|c|c|c|c|c|c|c|c|c|c|c|c|c|c|}
\hline \multirow[t]{3}{*}{$\mathbf{N}$} & \multirow{3}{*}{ REACTIVOS } & \multicolumn{6}{|c|}{ MARCO } & \multicolumn{6}{|c|}{ ACOLLA } & \multicolumn{6}{|c|}{ TUNAN MARCA } \\
\hline & & \multicolumn{2}{|c|}{ NUNCA } & \multicolumn{2}{|c|}{$\begin{array}{l}\text { ALGUN } \\
\text { AS } \\
\text { VECES }\end{array}$} & \multicolumn{2}{|c|}{$\begin{array}{l}\text { SIEMPR } \\
\text { E }\end{array}$} & \multicolumn{2}{|c|}{ NUNCA } & \multicolumn{2}{|c|}{$\begin{array}{l}\text { ALGUNAS } \\
\text { VECES }\end{array}$} & \multicolumn{2}{|c|}{ SIEMPRE } & \multicolumn{2}{|c|}{ NUNCA } & \multicolumn{2}{|c|}{$\begin{array}{l}\text { ALGUNAS } \\
\text { VECES }\end{array}$} & \multicolumn{2}{|c|}{ SIEMPRE } \\
\hline & & $\mathbf{N}$ & $\%$ & $\mathbf{N}$ & $\%$ & $\mathrm{~N}$ & & $\mathrm{~N}$ & $\%$ & $\mathrm{~N}$ & \% & $\mathrm{N}$ & $\%$ & $\mathrm{~N}$ & & $\mathrm{~N}$ & & $\mathrm{~N}$ & \\
\hline 1. & $\begin{array}{l}\text { Cuando se molesta tu esposo te } \\
\text { insulta. }\end{array}$ & 3 & 10 & 20 & 67 & 7 & 23 & 11 & 36 & 14 & 47 & 5 & 17 & 10 & 33 & 15 & 50 & 5 & 17 \\
\hline 2. & $\begin{array}{l}\text { Te grita por el modo en que haces } \\
\text { tus tareas }\end{array}$ & 14 & 47 & 16 & 53 & 0 & 0 & 22 & 73 & 08 & 27 & 0 & 0 & 20 & 67 & 10 & 33 & 0 & 0 \\
\hline 3. & $\begin{array}{l}\text { La acusa de causar conductas } \\
\text { violentas. }\end{array}$ & 15 & 50 & 13 & 43 & 2 & 7 & 25 & 83 & 05 & 17 & 0 & 0 & 18 & 60 & \begin{tabular}{|l|}
10 \\
\end{tabular} & 33 & 2 & 7 \\
\hline 4. & Te crítica sobre tu apariencia & 28 & 93 & 2 & 7 & 0 & 0 & 29 & 97 & 1 & 3 & 0 & 0 & 25 & 83 & 5 & 17 & 0 & 0 \\
\hline
\end{tabular}

Fuente: Cuestionario aplicado por las investigadoras a los padres e hijos de Marco en Mayo del 2015

En el cuadro $\mathrm{N}^{\circ} 02$ se percibe la violencia verbal conyugal en los distritos de Marco, Acolla y Tunanmarca, donde los porcentajes más altos demuestran que nunca han sufrido violencia; en Marco del 50\% al 93\%,en Acolla es del $47 \%$ al $97 \%$, y en Tunanmarca del $50 \%$ al $83 \%$, sin embargo de acuerdo a la opinión de las propias encuestadas algunas veces sufrieron violencia en Marco 7\% al 47\%, en Acolla del 3\% al 36\% y en Tunanmarca del $17 \%$ al $33 \%$ y siempre han sufrido violencia representa los porcentajes más bajos como es en Marco del 3\% al 23\% , en Acolla 17\% y en Tunan Marca del 3\% al 17\% . La violencia verbal hacia la mujer del parte del cónyuge se manifiesta por insultos, gritos, acusaciones, críticas y amenazas, por lo general ocurre solo algunas veces.

Tabla 3. Violencia psicologica conyugal

\begin{tabular}{|c|c|c|c|c|c|c|c|c|c|c|c|c|c|c|c|c|c|c|c|}
\hline \multirow[t]{3}{*}{$\mathrm{N}^{0}$} & \multirow[t]{3}{*}{ REACTIVOS } & \multicolumn{6}{|c|}{ MARCO } & \multicolumn{6}{|c|}{ ACOLLA } & \multicolumn{6}{|c|}{ TUNANMARCA } \\
\hline & & \multicolumn{2}{|c|}{ NUNCA } & \multicolumn{2}{|c|}{$\begin{array}{l}\text { ALGUN } \\
\text { AS } \\
\text { VECES }\end{array}$} & \multicolumn{2}{|c|}{$\begin{array}{l}\text { SIE } \\
\text { MPR } \\
\text { E }\end{array}$} & \multicolumn{2}{|c|}{ Nunca } & \multicolumn{2}{|c|}{$\begin{array}{l}\text { ALGUN } \\
\text { AS } \\
\text { VECES }\end{array}$} & \multicolumn{2}{|c|}{$\begin{array}{l}\text { SIEMP } \\
\text { RE }\end{array}$} & \multicolumn{2}{|c|}{$\begin{array}{l}\text { NUNC } \\
\text { A }\end{array}$} & \multicolumn{2}{|c|}{$\begin{array}{l}\text { Alguna } \\
\text { s veces }\end{array}$} & \multicolumn{2}{|c|}{ siempre } \\
\hline & & $\mathrm{N}$ & $\%$ & $\mathbf{N}$ & $\%$ & $\mathbf{N}$ & $\%$ & $\mathrm{~N}$ & $\%$ & N & $\%$ & N & $\%$ & $\mathbf{N}$ & $\%$ & $\mathbf{N}$ & $\%$ & $\mathrm{~N}$ & $\%$ \\
\hline 1. & $\begin{array}{l}\text { Te sientes humillada por el } \\
\text { maltrato de tu pareja. }\end{array}$ & 15 & 50 & 12 & 40 & 3 & 10 & 20 & 66 & 08 & 27 & 2 & 7 & 18 & 60 & 10 & 33 & 2 & 7 \\
\hline 2. & Te amenaza con golpearte. & 20 & 67 & 7 & 23 & 3 & 10 & 15 & 30 & 13 & 43 & 2 & 7 & 8 & 26 & 20 & 67 & 2 & 7 \\
\hline
\end{tabular}

Fuente: Cuestionario aplicado por las investigadoras a los padres e hijos de Marco en Mayo del 20 I5

En el cuadro $\mathrm{N}^{\circ} 03$ se observa la violencia psicológica conyugal, que nunca han sufrido violencia en Marco, oscilando del 50\% al 67\%; en Acolla del 30\%al $66 \%$ y en Tunanmarca del $26 \%$ al 60\%. Lo que nos demuestra los resultados es que el mayor porcentaje no sufren violencia en Acolla; algunas veces sufren violencia en Marco del 23\% al 40\%, en Acolla del 27\% al 43\% y en
Tunan marca del 33\% al 67\% y aquellos que siempre han sufrido violencia de parte de su conyugue en Marco se tiene un porcentaje del 10\%; en Acolla el 7\% y en Tunan Marca es similar 7\%. En concreto los que sufren siempre violencia psicológica son solo unos casos, siendo mayor en Marco, seguido por Acolla y Tunamarca que tiene el mismo resultado. 
La violencia psicológica hacia la mujer del parte del cónyuge ocurre algunas veces y se manifiesta a través de humillaciones y amenazas.

Tabla 4. Violencia sexual conyugal

\begin{tabular}{|c|c|c|c|c|c|c|c|c|c|c|c|c|c|c|c|c|c|c|c|}
\hline \multirow[t]{3}{*}{$\mathrm{N}^{0}$} & \multirow[t]{3}{*}{ REACTIVOS } & \multicolumn{6}{|c|}{ MARCO } & \multicolumn{6}{|c|}{ ACOLLA } & \multicolumn{6}{|c|}{ TUNANMARCA } \\
\hline & & \multicolumn{2}{|c|}{ NUNCA } & \multicolumn{2}{|c|}{$\begin{array}{l}\text { ALG } \\
\text { UNAS } \\
\text { VECE }\end{array}$} & \multicolumn{2}{|c|}{$\begin{array}{l}\text { SIE } \\
\text { MPR } \\
\text { E }\end{array}$} & \multicolumn{2}{|c|}{ Nunca } & \multicolumn{2}{|c|}{\begin{tabular}{|l} 
ALGUN \\
AS \\
VECES \\
\end{tabular}} & \multicolumn{2}{|c|}{$\begin{array}{l}\text { SIE } \\
\text { MPR } \\
\mathrm{E} \\
\end{array}$} & \multicolumn{2}{|c|}{ NUNCA } & \multicolumn{2}{|c|}{$\begin{array}{l}\text { Alguna } \\
\text { s veces }\end{array}$} & \multicolumn{2}{|c|}{ siempre } \\
\hline & & $\mathbf{N}$ & $\%$ & $\mathbf{N}$ & $\%$ & $\mathrm{~N}$ & $\%$ & $\mathbf{N}$ & $\%$ & $\mathbf{N}$ & $\%$ & $\mathrm{~N}$ & $\%$ & $\mathrm{~N}$ & $\%$ & $\mathbf{N}$ & $\%$ & $\mathbf{N}$ & $\%$ \\
\hline 1. & $\begin{array}{l}\text { Te ha exigido tener relaciones } \\
\text { sexuales. }\end{array}$ & 30 & 100 & 0 & 0 & 0 & 0 & 30 & 100 & 0 & 0 & 0 & 0 & 30 & 100 & 0 & 0 & 0 & 0 \\
\hline 2. & $\begin{array}{l}\text { Han usado la fuerza física para } \\
\text { tener relaciones sexuales. }\end{array}$ & 30 & 100 & 0 & 0 & 0 & 0 & 30 & 100 & 0 & 0 & 0 & 0 & 30 & 100 & 0 & 0 & 0 & 0 \\
\hline 3. & $\begin{array}{l}\text { Su pareja le obliga a hacer } \\
\text { alguna actividad sexual que }\end{array}$ & 30 & 100 & 0 & 0 & 0 & 0 & 30 & 100 & 0 & 0 & 0 & 0 & 30 & 100 & 0 & 0 & 0 & 0 \\
\hline
\end{tabular}

Fuente: Cuestionario aplicado por las investigadoras a los padres e hijos de Marco en Mayo del 2015

En el cuadro $\mathrm{N}^{\circ} 04$ se muestra que el 100\% de personas encuestadas respondió que su cónyuge nunca le ha exigido a tener relaciones sexuales en Marco, Acolla y Tunanmarca, por otro lado el 100\% también menciona que su cónyuge nunca ha usado la fuerza física para tener relaciones sexuales con ella, y por último el 100\% manifiesta nunca haber sido obligada a hacer alguna actividad sexual que ella considere humillante o desagradable.

A continuación se presenta algunas entrevistas realizadas, a fin de evidenciar cómo se manifiesta la violencia a la mujer de parte del cónyuge.

Tabla 5. Violencia de padres e hijos en los distritos de marco, Acolla y Tunanmarca

\begin{tabular}{|c|c|c|c|c|c|c|c|c|c|c|c|c|c|c|c|c|c|c|c|}
\hline \multirow[t]{3}{*}{$\mathrm{N}^{\mathrm{O}}$} & \multirow[t]{3}{*}{ TIPOS DE VIOLENCIA } & \multicolumn{6}{|c|}{ MARCO } & \multicolumn{6}{|c|}{ ACOLLA } & \multicolumn{6}{|c|}{ TUNANMARCA } \\
\hline & & \multicolumn{2}{|c|}{ NUNCA } & \multicolumn{2}{|c|}{$\begin{array}{l}\text { ALGUNAS } \\
\text { VECES }\end{array}$} & \multicolumn{2}{|c|}{ SIEMPRE } & \multicolumn{2}{|c|}{ Nunca } & \multicolumn{2}{|c|}{$\begin{array}{l}\text { ALGUNA } \\
\text { S } \\
\text { VECES } \\
\end{array}$} & \multicolumn{2}{|c|}{ SIEMPRE } & \multicolumn{2}{|c|}{ NUNCA } & \multicolumn{2}{|c|}{$\begin{array}{l}\text { ALGUNA } \\
S \\
\text { VECES }\end{array}$} & \multicolumn{2}{|c|}{ SIEMPRE } \\
\hline & & N & $\%$ & $\mathrm{~N}$ & $\%$ & $\mathbf{N}$ & $\%$ & $\mathbf{N}$ & $\%$ & $\mathbf{N}$ & $\%$ & $\mathrm{~N}$ & $\%$ & $\mathbf{N}$ & $\%$ & $\mathrm{~N}$ & $\%$ & $\mathbf{N}$ & $\%$ \\
\hline 1. & $\begin{array}{l}\text { Violencia verbal(gritos, } \\
\text { insultos) }\end{array}$ & 20 & 97 & 1 & 3 & 0 & 0 & 28 & 97 & 2 & 3 & 0 & 0 & 24 & 80 & 6 & 20 & 0 & 0 \\
\hline 2. & $\begin{array}{l}\text { Violencia física(golpes, } \\
\text { moretones) }\end{array}$ & 28 & 97 & 2 & 3 & 1 & 3 & 23 & 76 & 7 & 24 & 0 & 0 & 20 & 66 & 10 & 34 & 0 & 0 \\
\hline 3. & $\begin{array}{l}\text { Violencia psicológica } \\
\text { (amenazas, }\end{array}$ & 20 & 66 & 10 & 34 & 0 & 0 & 29 & 97 & 1 & 3 & 0 & 0 & 24 & 80 & 5 & 17 & 1 & 3 \\
\hline 4 & $\begin{array}{l}\text { Violencia } \\
\text { sexual(relaciones }\end{array}$ & 30 & 100 & - & - & - & - & 30 & 100 & - & - & - & - & 30 & 100 & - & - & - & - \\
\hline
\end{tabular}

En el cuadro $N^{0} 5$ se observa que los porcentajes más altos nos señalan que no existe nunca violencia verbal de padres a hijos, en Marco oscila el 97\%, que no hubo violencia verbal y que tuvieron solo algunas veces 3\%. En Acolla es lo mismo $97 \%$ y 3\% algunas veces. En Tunanmarca el $80 \%$ respondieron que no hubo violencia verbal y que algunas veces sufrió solo el $3 \%$; seguido por la violencia física nunca en Marco 97\%, algunas veces $3 \%$; en Acolla $76 \%$ nunca y algunas veces $24 \%$, en Tunanmarca $66 \%$ nunca hubo violencia de padres a hijos y el 34\% algunas veces. La violencia psicológica de padres a hijos nunca sucedió en Marco 66\%, algunas veces 34\%; en Acolla no sufrieron nunca violencia psicológica en un 97\%, algunas veces el 3\%; en Acolla nunca sufrieron 97\%, algunas veces $3 \%$ y Tunan marca nunca $97 \%$ y algunas veces sufrieron violencia de padres a hijos $80 \%$ y $17 \%$ algunas veces y $3 \%$ siempre sufren violencia psicológica. Finalmente en cuanto a la violencia sexual manifestaron no tener en los tres distritos.

La violencia de padres e hijos en los distritos de Marco, Tunan Marca y Acolla ocurre algunas veces para corregir a los hijos en su desarrollo personal y se manifiesta, principalmente a través de la violencia verbal, seguido por el físico y psicológico. No se tiene problemas de violencia sexual.

\section{Discusión}

La violencia física en la mujer de parte de cónyuge de Acolla, Marco y Tunan Marca se manifiesta a través de patadas, sacudidas, jaloneos, empujones y moretones y ocurre de parte de sus parejas. 
Estos resultados se sustentan en:

BARTRA; NÚÑEZ y RAMOS (2010) en el estudio "Consecuencias de la violencia doméstica en la mujer", cuando manifiestan "que el 88,6\% sufre violencia física: los mayores problemas de salud manifestados son los hematomas y molestias estomacales. Así mismo se sustenta en Santa Cruz (2007) en la investigación "Violencia Familiar Tipo Física Contra la Mujer": Prevalencia y Características Sociodemográficas. División Clínico Forense, Instituto de Medicina Legal y Ciencias forenses cuando exponen "que durante el año 2007 se registró 7.340 reconocimientos médicos legales: del cual el 75.45\% corresponde a la violencia física. La gran mayoría de mujeres víctimas de violencia familiar de tipo físico se encuentran en el grupo etario de las mujeres adultas entre los 25 y 60 (73,3\%)".

Por otra parte, se sustenta en Gonzales (2009) en su investigación "Hacia la unificación e integración del sistema de protección frente a la violencia familiar" presenta un resumen de las estadísticas del Poder Judicial, cuando dicen que "el número de denuncias por violencia familiar según el Sistema de Información de Apoyo al Trabajo Fiscal (SIATF) del Ministerio Público fue 5.690 en el año 2007: 54,23\% por violencia física y 50,67\% por violencia psicológica. En el año 2008 ingresaron 5.064: $45,77 \%$ por violencia física y $49,32 \%$ por violencia psicológica".

Además, se presenta los resultados de los casos de entrevistas realizadas, de Lucia quien manifestó lo siguiente: "En un primer momento los primeros meses de convivencia estábamos todo bien, el problema empezó cuando nació mi bebe ya no tenía apoyo de mis padres, mi esposo no terminaba estudiar la universidad por lo que empezó las discusiones porque no teníamos dinero para nuestra necesidades. A partir de allí inicio nuestras peleas, la primera vez que me pego a patadas fue cuando le reclame que debería trabajar". La violencia verbal a la mujer de parte del cónyuge se manifiesta a través de insultos, gritos, acusaciones y críticas estos resultados se respaldan en las siguientes entrevistas:

Lucia: “. . Cuando se le preguntó, si su esposo le agrede en forma verbal; ella respondió diciendo si mi esposo es bueno pero cuando se molesta de algo o se comporta mal mis hijos mi esposo me grita, me insulta feamente, diciendo, que es por mi culpa, porque yo les consiento según él, pero no es así yo les corrijo a mis hijos. Estos insultos es casi siempre por cualquier cosa, me insulta a veces pienso en separarme pero no lo hago por mis hijos..."

Felicitas: " Cuenta que nunca le golpea su esposo, dice yo no conozco cómo será el maltrato de un hombre; vivo bien solo que cada vez que se emborracha me dice de todo me insulta me grita, me dice que soy analfabeta, bruta, ignorante, no sabes nada y todas las palabras habidas en su boca y grita tan fuerte que todos los que están cerca escuchan..."

La violencia psicológica a la mujer de parte de su cónyuge en Acolla, Marco y Tunan Marca se manifiesta algunas veces a través de amenazas y humillaciones. Estos resultados se sustentan Labrador, Paz y Fernández (2004) cuando manifiestan; "que las victimas sufren intimidación, desvalorización, sentimientos de culpa o sufrimientos, humillaciones, descalificaciones o ridiculizaciones tanto en público como en privado..." por otra parte se sostiene en Lestonnac (2009) Línea de Base del Proyecto «Participación Comunitaria para prevenir La prevalencia de la Violencia Familiar" "cuando dice que la más alta prevalencia de violencia se presenta en la zona urbana del distrito de Guadalupe, donde el 63\% de las mujeres en edad fértil ha sufrido algún tipo de violencia psicológica..."

La violencia sexual a la mujer de parte de su cónyuge en Acolla, Marco y Tunan Marca no se manifiesta nunca a través de forzamientos. Así mismo nunca han cedido a los requerimientos sexuales de algún familiar, en lo que respecta a las entrevistas solo un caso manifiesta que algunas veces su esposo le pegaba para tener relaciones sexuales forzadas. Estos resultados se manifiestan en Machuca y Velarde (2009) de la ruta crítica del sistema policial - judicial en los Casos de Violencia Familiar en los Distritos de San Juan de Miraflores, Villa El Salvador y Villa María del Triunfo cuando dicen "...que los operadores policiales confirmaron que las mujeres no denuncian los casos de violencia sexual por vergüenza. En otros casos entienden que este tipo de agresión no amerita una denuncia...". Por otra parte se sustenta en Labrador, Paz, de Luis y Fernández - Velasco (2004) cuando dice; "....Este tipo de violencia es más difícil de ubicar en especial si se disfraza de atención o preocupación por la víctima...". La violencia de padres a hijos ocurre algunas veces para corregir a los hijos en su desarrollo personal y se manifiesta principalmente a través de la violencia verbal (grito e insultos, seguido por la violencia física y psicológica y no se ha tenido problemas de violencia sexual.

Estos resultados se respaldan en, Aliaga (2015) cuando dice que el maltrato infantil es considerado en nuestro medio como elemento determinante en la formación integral de los niños, estos condicionantes empleados son los personales transmitidos generacionalmente de padres a hijos y los socios culturales expresados en la práctica naturalizada de la violencia como una forma de educar o corregir faltas.

\section{Conclusiones}

I. La violencia física a la mujer de parte del cónyuge en Acolla, Marco y Tunan Marca es algunas veces y se manifiesta a través de golpes, patadas, sacudidadas y/o jaloneos, empujones, originado por el consumo de 
alcohol, celos y por situación económica.

2. La violencia verbal a la mujer de parte del cónyuge ocurre algunas veces y se manifiesta a través de gritos e insultos.

3. La violencia psicológica a la mujer se manifiesta a través de humillaciones y amenazas, solo algunas veces.

4. La violencia sexual a la mujer en Marco, Acolla y Tunan Marca no se da manifiesta según los cuestionarios aplicados, sin embargo en las entrevistas se ha detectado un solo caso en Marco.

5. La violencia de padres a hijos se manifiesta solo algunas veces, principalmente a través de la violencia verbal, seguida por la violencia física y psicológica. Y no existe la violencia sexual.

6. La violencia familiar en los distritos de Acolla, Marco y Tunan Marca se manifiesta a través de la violencia física (golpes y patadas), violencia verbal (insultos), violencia psicológica (Humillaciones) y en cuanto a la violencia sexual manifiestan no tenerlos a excepción de un solo caso. Así mismo los factores que originan la violencia familiar son debidos al consumo de alcohol, a los celos y por la necesidad económica.

\section{Referencias}

Alvarado Z. G., Salvador M. J., Estrada y Terrones G. A. (1998) "Prevalencia de violencia doméstica en la ciudad de Durango" México. Recuperado de

www.redalyc.org/articulo.oa?id= 0640604

Aliaga R.; (2015) "Maltrato infantil como elemento de formación integral en los niños de las familias en el barrio de Azana Chupaca". Tesis - UNCP.

Araujo, R. Vargas y Velarde, C. - (2009) "Evaluación de la Ruta Crítica del Sistema Policial"- Judicial en los Casos de Violencia Familiar en Los Distritos de San Juan de Miraflores, Villa El Salvador y Villa María Del Triunfo.

Bartra P. V., Núñez J., y Ramos A. (20 I0). "Consecuencias de la violencia doméstica en la mujer", Ministerio de la Mujer y Poblaciones Vulneradas. Recuperado de : www.mimp.gob.pe/files/.../estado_investigaciones2006-20l0.pdf

Cordero G. A. (2012) Violencia familiar y su influencia en el rendimiento académico de los alumnos de educación secundaria de la I.E. "Uniciencias "del distrito de Independencia. Lima - Perú.

Fondo de la Naciones Unidas para la Infancia - UNICEF (2008). Base Educativa y Comunitaria d Apoyo
BECA "Estudio sobre maltrato infantil en el ámbito familia" Asunción Paraguay.

Labrador F.J., Rincón G. P., Fernández V. R. (20 I0). Mujeres Víctimas de la Violencia Domestica Programa de actuación Madrid: Ediciones Pirámide.

López L.(20 I I) Violencia hacia la mujer por su pareja; intervenciones orientadas a su manejo integral, Tesis Universidad de Ciencias Medicas de 100 fuegos. 